\title{
The paradigm-shift toward the next generation of drugs
}

Sara Diani ${ }^{1}$, Claudio Lombardo²

1 Université Européenne Jean Monnet, "Giovanni Ferrari" School of Specialization in Music

Therapy, Padua, Italy

2 Independent researcher, Via Maso della Pieve 27, Bolzano, Italy

Corresponding author: Sara Diani

dr.saradiani@gmail.com

$+393294642536$

ORCID: 0000-0003-3079-0325

Keywords: cybernetics, complex systems, new drug development; human system 


\begin{abstract}
Specialized, reductionist, and linear approaches are applied in clinical research; they are based on linear logic and used towards therapeutic molecule-based targets. However, those approaches do not consider a systemic vision that describes the remote cause of the pathogenic activation. We propose new theoretical and practical methods for the next drug generation development. Self-organization, network structure, hierarchical organization, non-linearity, feedback circuits, reactions to information, and the view of drugs as information clarify the existing pharmacological methods. We suggest a perspective and hierarchical vision of the human organism based on six levels (mechanic and structural; metabolic; bodily dynamic; emotional, cognitive, spiritual). The therapy should restore the self-organization of every level using the "intelligent" modulation of the network responses. Multi-targeted drugs should act on the remote cause of the pathogenic cascade and be administered based on personal variability and networks. This approach may help the development of individualized, precise, and integrated medicine.
\end{abstract}




\section{Introduction}

Specialized, reductionist, and linear approaches are extensively used in medical and pharmaceutical research. Reductionist systems understand the mechanisms of many diseases and the receptors involved in pathogenic cascades. These systems are based on linear logic and become the therapeutic targets towards new molecule discovery. Nevertheless, the linear logic allows the development of molecules that do not describe the remote cause of the pathogenic activation. Finding the upstream cause of pathogenic events requires a systemic vision. A novel paradigm of network medicine [1-3] and precise/individualized medicine [4-6] is emerging. The World Health Organization (WHO) guidelines [7] indicates a new concept defined as integrated medicine. Integrated medicine focuses on non-conventional disciplines, indirectly based on cybernetics and complex systems. Cybernetics and complex system allow a systemic perspective to understand chronic and comorbid illnesses.

In this article, we will propose new theoretical and practical methods for the development of the next drug generation and future medicine. Our aim is to create a bridge between systemic and complex reductionist epistemologies.

\section{Premises - Cybernetics and complex systems}

\subsection{Cybernetics}

Cybernetics and complex systems are complementary disciplines that involve epistemology. Epistemology studies the human organism from a perspective and logical point of view in the context of the theory and medical practice. 
The term Cybernetics indicates the study of the auto-regulation and communication phenomena, as well as the theory of messages (theory of information) and messages of commands. A command is the sending of messages that modify the behavior of who/what receives them [8].

An initial, accurate definition defines cybernetics as the discipline that concerns the united study of processes regarding communication and control within the animal and a machine [9]. A "second cybernetics," according to Bateson, describes the human condition without relating it back to a machine. The fundamental data that emerges from Bateson's epistemology [10] is that "no one part of this interacting system can exert univocal control over the rest of the system or over any other component of the system". Every time that this rule is violated, an epistemological error is committed.

Cybernetics is based on the concept of the system. A system is an object that consists of other objects called subsystems [11]. Subsystems are systems composed of other subsystems. A system is a group of interacting elements. What's interesting about a system are the relations and operations between the elements, more than the elements themselves [12]. One can study the connection between the "system" and "subsystem", which reflects a defined relationship between the objects [11].

The fundamental bases of a system are auto-regulation, communication, and adaptation [9]. The second crucial concept of cybernetics is "the state" of a system (or subsystem). The states are different configurations or behaviors of a system at a given time. Like the concept of a system, the concept of a state is a hidden relationship: the relationship between two moments in time.

Cybernetics studies the organization of systems in space and time, how subsystems are connected to the system and how their change in state may influence the state of other subsystems. Intentional organization in time is called control. 
We can expand the definition of cybernetic and complex systems to a set of elements interacting amongst themselves, in the form of an exchanging of materials, energy, and information, which is translated into communication. Communication, signals, information, and feedback are some of the central concepts of cybernetics and complex systems.

In a system, the action of an element on another implies reaction of the second element towards the first; this is called the feedback loop. Positive loops amplify tendencies; they create and reproduce. Negative loops reduce tendencies and bring the system back to its apparent equilibrium. A cybernetic system is equilibrated. It regulates itself and requires enough negative loops to respond to the positive ones; it obtains auto-stability and auto-adaptivity from the environment [13]. In cybernetics, the principle of required variety allows a system to acquire or construct adequate information about the environment [14].

Bateson used the concept of feedback ('reaction') to study autocorrection and recursive processes in living systems.

\subsection{Complexity and complex systems}

The science of complexity describes evolutive processes of complex systems, understood as a) a composition of many interdependent parts b) that interact with each other through a sort of competitive, non-linear collaboration and c) that bring self-organized, emergent behaviors [15]. There are different definitions of complex systems based on different systemic properties [16-18]. Today, "complexity" can be defined as an interpretive framework of the natural and social sciences. It is an approach or a process more than a hypothesis or theory. It takes note of epistemological and ontological insufficiencies of reductionism [19]. 
A complex and adaptive system is a set of free individual agents acting in unpredictable ways and whose dynamic modifications are so interconnected that the action of one factor changes the context for all the other ones $[11,20]$.

Every living organism is a Complex Adaptive System (CAS) or equally defined as CS (Complex System). Human beings, groups of human beings, immune systems, the process of elaborating thoughts, and education are CSs [9, 21].

We can define a human as a CS in which everything that enters into a flow-through system interacts with it and induces a dynamic self-regulation response [13]. The CS can maintain its fundamental, internal, dynamic equilibrium while it is also undergoing various processes of change [22]. Complex systems are characterized by relationships between heterogeneous parts that reciprocally interact in a defined space; they possess an organization that is both horizontal and hierarchical, with many types of intersecting interactions. They are characterized by continuous adaptation of single part evolution. In addition, they show movement instabilities and bifurcation phenomena (in which a small variation in parameter values can cause a qualitative or topological change in the system) [23]. The same deterministic aspect rises a great variety of dynamic possibilities, so-called "deterministic chaos" [24, 25]. The complex system dynamics are very far from thermodynamic equilibrium [26]. They work in non-linear conditions, amplifying small disruptions in a disproportionate way and creating new, unpredictable dynamics. They do not obey linear causality (or sequential). Linear causality proposes a direct derivation of one effect from one or more numerically determinable and predictable causes [27]. Non-linear causal inferences, however, are reciprocal influences between the components structured as a network. More than causes or direct consequences, these non-causal inferences are different subsystems influenced by each other in a reciprocal and circular way. The CS produces self-organized forms and emergent phenomena, not 
explainable by the observation of any one single part and comprehensible only in a holistic, not reductionist view [28].

The level of complexity of a system depends on its number of components and the number and direction of interactions [29].

Emergent behavior emerges from the linear and non-linear interactions between the components themselves [30, 31]. Emergence makes the system displaying macroscopic properties that are difficult to predict based on the laws that govern their components taken individually. Above all, it is the emergent behavior that requires the integration of reductionism with complexism.

Behavioral attractors (temporal or trajectory evolutions) play a considerable role $[32,33]$ and "capture" the emerging properties of the system [27]. They bring the system towards the emergence of a form that is never premeditated and created due to its self-organizational capacity. To comprehend the individual behavioral attractors, one should better understand the state of the system and its probable evolutions. This would allow the design of consequent therapies.

The other fundamental property of CSs is dynamism (evolution, adaptation). The system adapts its behavior to avoid errors and thus to learn from its mistakes. The system optimizes the answers to adapt itself to the environment, learning continuously from it. Through adaptation and heuristic learning the best possible responses to stimuli are selected [34].

\section{The human system}

We can define a human as an open system, able to exchange material, information, and energy with the environment in order to maintain its organization, to win out against the degradation of time and to turn towards a better complexity $[35,36]$.

This also implies that humans can self-heal in the presence of efficient stimuli. This is achieved through a dynamic modality based on the exchange of information between the various parts of the 
system. An example can be the extracellular space and the extracellular matrix (ECM) [37-39]. These are integrated and interconnected by a field on which all these activities are carried out. Instead of homeostasis, it is more correct to talk about calibration [12]: fluctuations reach a certain limit until the deviation cannot be corrected (feedback).

In practice, this property of complex biological systems is tied to the network of interconnection between cybernetically organized molecules of the system. The complex systems are subjected to three fundamental principles:

1) conformity to the goal (the entire cybernetic system is placed under the control of the vegetative command system);

2) holographic principle (every component of the system is informed of how much is happening in the context of the entire organism: everything acts on everything);

3) synergism principle (each dynamical phenomenon is connected to another ones) [13].

\subsection{Information and adaptation}

As reported by the Stanford philosophical encyclopedia [40], there is quantitative and qualitative information. In this paper, we will cover only qualitative information. A qualitative information is a scheme of a structure that can be adopted, reproduced or used to make decisions [41]. Information is message/sign (acknowledged even at a distance) or memory (because can be stable over time). Based on these concepts, the system develops a global, organized response, that is much larger in magnitude than the entity of the information itself. The complex system reacts to stimuli in a disproportionate, exponential way, and completely restructures itself. Consequently, when the response is completed, it will try to limit the ampliative modifications through feedback.

Drugs and therapies are types of information currently studied in a linear way. The actual strategy measures the direct effect of a drug on one or more receptors, observing therapeutical and collateral 
effects. In this way, the effectiveness of a drug is assessed in an average time and in a limited area. However, the drug activates a complex cascade in the system and provokes unpredictable bifurcations. The collateral effects are the results of the passive and active influence of the system itself. For instance, we do not ever consider new symptoms or illnesses that appear 10 years from now, as a side effect of the drug. But, because of the inferential relationships and continuous evolution, these new symptoms could also be related to the drug itself in an indirect way. Recognizing these phenomena, we argue that drugs and therapies should be studied in a perspective and logical way that considers the principles of non-linearity, systemic response, adaptation, calibration through negative feedback, evolution, and appearance of phenomena emerging through time. Another crucial concept to account for new drug development is the entropy. The dynamic equilibrium of a system can be altered by entropy. The information that demonstrates a network of advantageous possible responses to the system, represents a negative entropy [42]. It is possible to diminish the entropy in a system by augmenting the received information, through more detailed measures of its configuration. We are convinced that from the therapeutic point of view, the best therapeutic solution (the information) should maintain the least amount of entropy possible. The information compatible with systemic rules, and that demonstrates a network of energetically advantageous possible responses to the system, represents a negative term in the entropy of a system. Thus, one can define information as negative entropy [42]. The natural place of complexity is between entropy and information [15].

The process of receiving and using information is identified with our process of adaptation to the external environment, and of living in an effective way in it [43]. It is complementary and successive to the transmission of information [8]. The dynamic equilibrium of the organism is altered when it receives a new piece of external information. At this point either the system reinstates the preceding equilibrium, or it configures itself into a new structure, different from what 
came before. It generates a new organization and dissipating energy [44]. Entropy measures the lack of information in the effective structure of the system [42, 45]. The system always tries to maintain the most basic entropy possible, taking it from the environment.

Therefore, from the therapeutic point of view, the best information possible is that which helps the organism maintain the least amount of entropy possible. Information that does not block its processes but modulates them in an efficient manner. In the next sections, we will propose a new complex logic that aims at the development of a pharmacological activity based on all the above factors.

\section{The hierarchical organization of the human organism}

Our complex system has a network-like organization that acts on many levels [46]. We can identify several networks (e.g. molecular, tissue or even cultural networks). In this article, we will focus on system networks that interact amongst themselves. We propose a perspective and hierarchical vision of the human organism that could be used to understand the susceptibility of the organism itself, the illness structure, and the type of therapeutic information possible to administer. We suggest a structured approach to the development of integrated, individualized and precise medicine based on systemic logic. This logic is complex, circular and multifaceted. Understanding systemic logic permits having a philosophical, epistemological, and scientific vision. This allows the harmonization of the therapeutic disciplines with all the individual therapeutic courses. Our new hypothesis of progressive, hierarchical organization is based on six levels of the system. The premise is that every level of a system influences the surrounded levels in a circular way. This is done through positive and negative feedbacks. The foundation of our approach is the somatopsychic origins of the majority of human processes. These processes are based on body and brain, which have a coordinated activity. 
We present a monist vision of body and mind. In fact, the brain emerges from the body and is in the body. Consequently, it is from the body that thoughts arise, just like at the foundation of everything rational, you can find something irrational [47]. It is only after the process of intuition (that can occur in corporeal feelings), through settling, ramification, and elaboration, that the order and the coherence of superior cognitive functions appear. The mind and its processes emerge from preceding levels. This is possible because there is cognition in every perception and in every cellular and organic activity [36].

The organizational systematic levels, going from the lowest to the highest in ascending order, are (Fig. 1) [48]:

- Mechanic and structural. Here are the musculoskeletal system and all the sustaining structures.

- Metabolic. On this level, we find the gastrointestinal and endocrine systems, which process foods that we ingest and transform them into energy for the body.

- Bodily Dynamic. Here, practically all the physiological and pathological processes enter on the physical level.

- Emotional. On this level, we analyze the physical and emotional sphere, which interact with both body and cognitive sphere to influence one another. Emotions are the contact points between the body and the intellectual activity.

- Cognitive: Cognitive processes characterize us as human beings. Here we find the neurological sphere. The emotional and mental levels are very difficult to separate because they work in a synergistic and complementary way. Usually, any problems in the psychological sphere are reflected onto the cognitive one, and vice versa.

- Spiritual: This represents our view of the world on a deep level. He who is spiritually active sees all the other levels as part of this last one. 


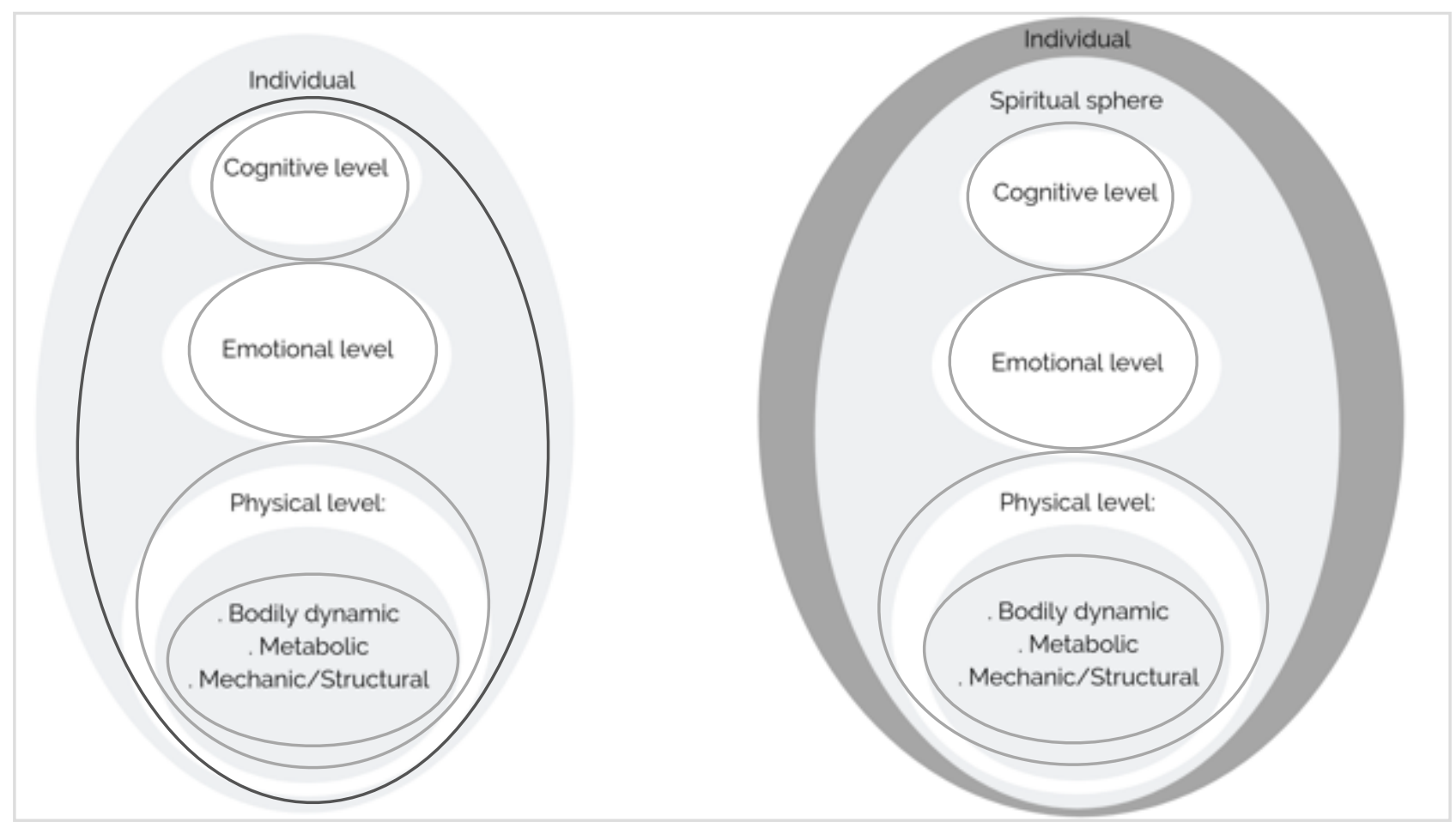

Figure 1: The six levels of the human hierarchy

The six levels are correlated amongst themselves and indivisible because they work, in the healthy individual, in full harmony. Every level of the human system is susceptible to different types of information from the most unrefined, as the mechanical force to the pharmaceutical molecules, to pure and non-material information in the guise of language or frequencies. The hierarchy structure, the holographic characteristics, the conformity to the goal, and the synergism of the levels trigger a systemic response. The information we administer should be compatible with the systemic answer so that the system is able to acquire and maintain the memory of the positive change that occurred after the treatment. Treatment that can thus be consolidated with a great efficiency and duration. There are many examples of reciprocal influence among the different levels, such as the bottom-up influence which is the influence of the body to the brain, and the top-down influence which is the 
influence of the brain to the body. Furthermore, we assess the bijective influence (communication between the body and the brain is bijective) and other organizational options of chronic illnesses.

\section{Types of influence among the different levels.}

\subsection{Bottom-up influence}

We report two examples of the bottom-up influence of the body to the brain.

1. Exercising through aerobic activity improves cerebral functions, such as neuroplasticity and the size of the hippocampus, or delays and alleviates the symptoms of Alzheimer's and Parkinson's [49-55]. Also, there are different studies on animal models that demonstrate an increase in the production of the neurotrophic factor derived from glial cell lines (GDNF) and from cerebral neurotrophic factor (BDNF), responsible for the formation of new synapses [56-58]. Beneficial states of body exercises have been observed in mice in terms of neuroprotection, neurogenesis in the dentate gyrus and improvement of symptoms of depression in Huntington and Parkinson's diseases [57, 59].

2. The microbiota-gut-brain connection. The microbiota is the set of bacteria that populate all surfaces of our body in contact with the external world. It is important to specify that microbiota is not a bacterial population that "falls" on an organism, but a population that grows and modifies itself according to the characteristics of the organism. Therefore, according to the actual and future research studies, the microbiota will serve as a set of indirect parameters for understanding other intrinsic characteristics of the organism. Among all the sub-communities of microorganisms, the most important is found in the 
intestine. It is the most important not only for the high number of bacteria, but also for the connections that the intestine has with endocrine, immune, and nervous systems [60]. Intestinal microbiota can influence many physiological parameters, including cognitive functions such as the learning process, the memory, and the decisional processes. The "stations" of this axis are microbiota-intestine-vagus nerve-brain. Additionally, the intestinal flora can alter the levels of circulating cytokines that could influence different brain functions $[61,62]$. The influence on the cerebral activity also occurs through the afferent branch of the vagus nerve [63] and the modulation of the tryptophan system [64], a precursor of the serotonin neurotransmitter. Up to now, some behavioral disorders have been connected to the intestinal flora such as anxiety, depression, and some cognitive disorders. However, it seems that the composition of the intestinal flora can play a role in other metabolic conditions that involve the central nervous system $[65,66]$. There is a possibility that intuitive decisional processes are based on interoceptive maps for visceral responses, which would allow the brain to make decisions based on intestinal activity [67].

\subsection{Top-down influences}

Now we will present two examples of the top-down influence of the brain to the body.

1. In times of stress, the brain can influence the composition of the intestinal flora [68] through the hypothalamic-pituitary-adrenal axis, which regulates the secretion of cortisol. This influences the immune activity of the cells, both on the local intestinal and the systemic levels. Cortisol can alter intestinal permeability and its barrier function. Consequently, it contributes to the modification of the composition of the intestinal microbiota [69]. In addition, stress alters numerous brain and bodily functions through 
the reduction of NK cells, the number of white blood cells, lymphocytes CD4+, IFN- $\gamma$, and a change in the leucocyte formula. It can also increase heart rate, blood pressure, regular antibodies of viruses present in latent phases in the organism and reduce nerve cells in the hippocampus. Stress can diminish the activity and efficiency of the immune system [70-72] and influence ageing [73]. Therefore, the point of view of a network is complementary to the top-down influence and, when integrated with classic reductionism, allows multiple and large steps forward.

2. Depression following a heart attack is a predictive death factor of a patient within the next 18 months [74]. No other parameter exists, cardiac or not, that has this ability to predict possible outcomes. Negative emotions, that are the basis of suffering, indicate negative effects on health [75]. On the contrary, positive emotions like happiness or optimism positively influence bodily functions. Among patients who underwent a coronary bypass, the most optimistic had a much faster recovery time with a smaller effect of complications during and after the surgery than those who were pessimistic [76].

\subsection{Bijective influence}

In most processes, communication between the body and the brain is bijective and concurrent.

1. Posture control. Thoughts formed in the brain can rise emotions that are then carried out through the body. For example, in a state of depression and sadness, the body curls in on itself, causing kyphosis and closure of the spine; these postural changes, in turn, influence the brain. Therefore, the body can change cerebral images and thus the substrate of thoughts. Modifying gestures, for example stretching the arms up, can 
modify the perception of a situation and one becomes more optimistic and positive; this occurs because the level of cortisol decreases, and testosterone levels increase.

2. Pain. During chronic pain, a continuous nociceptive stimulation occurs that provoke some neuroplastic modifications. These then go on to reinforce the neuronal network of responses [77], increasing the perception of the pain itself and inducing, in some cases, depression and other secondary ailments.

3. Research correlates some psychological discomforts, such as post-traumatic stress disorder (PTSD) [78-80] or depression [81] with higher levels of cortisol.

\subsection{Other organizational options in chronic illnesses}

The levels described here not always display equal intensity. One can observe an organism that is very sick from a mental standpoint but healthy from a physical one. Vice versa, a very sick person from the physical standpoint could be healthy from the mental or emotional one. For example, a cancer patient can be very serene, and calm, with emotional and mental clarity through which he sends powerful and effective messages to other people, perhaps encouraging them. An observation of a group of borderline patients has been carried out where, in the phases of remission of the psychiatric illness, they were allergic to pollen; when the psychiatric symptoms returned, the pollen allergy vanished [82].

\section{Possible innovative therapeutic instruments}

The maintenance of an organism's state of health depends a) on the structural integrity of the singular components at every level and b) on the communication between the different levels and the different components (the self-organization of complex systems). The goal of a therapy is to 
restore the ability of systemic self-organization. At the same time, we should try to develop therapies that influence every level in a rational and complementary way (perhaps taking advantage of their hierarchical positions). Relaxation techniques are an example of how one can introduce information/energy, reduce entropy, and involve more levels at a time. For example, autogenic training [83] performances on multiple levels. Its application ranges from cardiocirculatory to neurological and psychological disorders. Evidence has shown that autogenic training improved the health of people who have suffered from stress and depression [84] and displayed positive effects on cancer [85], anxiety disorders [86, 87] irritable bowel syndrome [88], migraines [84] and other impairments.

It is possible to develop therapeutic and pharmacological strategies that use the same systemic effects of autogenic training through an "intelligent" modulation of the network responses. Given the circular logic, the positive and negative feedback, and the influence of different networks, it is not always possible to understand the original cause of a disease in a reductionist fashion.

Consequently, the best approach is to focus on the molecular processes of the individual networks and the complex system in its entirety. The best way to increase the probability of success is to modulate the dynamics of communication and influence between the different levels. This is in contrast with the current medical paradigm that targets single receptor and one cascade mechanism. To put our proposed paradigm into practice, it would be necessary to understand what the most typical patterns of pathological networks are and consider individual differences that modify the signs and symptoms coming from similar networks. This will allow the development of precision medicine. Furthermore, it is important to test whether the psychological and mental levels are involved in a primary way. Helping the management of feelings and supporting negative thoughts become a form of prevention, given that these are determining risk factors in the development of chronic illnesses. Evidence showed that the success of clinical treatments increases when the 
psychological feature of patients is considered [84-87]. This can be achieved with training, information, and integrated therapies.

To implement the integrated medicine approach, one should study:

- the effect of different types of information on different levels;

- how to integrate those type of information in a strategic way.

\section{Understanding pharmacological dynamics and prospects}

Complex logic permits the development of a new therapeutic approach (pharmacological or not) and to understand reactions to drugs in a rational manner. If we accept these premises, we can state the following hypotheses:

- Non-linearity explains allergic reactions, hypersensitivity and all modifications induced by a drug.

- Calibration describes fluctuations reached in a limit until the deviation cannot be corrected (via feedback). Calibration through negative feedback defines drug tolerance, which requires a progressive increase in drug dosage to couple to different active principles for the same pathology.

- Calibration explains also why, once a drug is administered, the system tends to return to the original manifestation. This occurs because, at the end of the pharmacological effect, the system returns to the preceding behavioral attractor.

- Homeostasis, or dynamic equilibrium, explains why a drug is inefficient. Blocking the systemic response without stimulating self-organization makes the system return to the predrug condition. Furthermore, after drug administration, the system could develop new dynamics through new bifurcations. With the current approaches, we consider these 
bifurcations independent, but they may be indirectly related to the drug we have administered, even after years.

- Deterministic chaos explains a large amount of individual variability due to different signs, symptoms, and reactions to the same stimulus.

- Networks clarify that the drug (information) initiates a response exactly like other physiological and pathological environmental stimuli [34]. The study of the overlap among different pathogenic networks and different behavioral attractors could carry out a more targeted prevention based on individual risk factors.

- Organization and communication between sub-components of the system explain why a single drug cannot modify all pathogenic processes when there is more than one diagnosis at a time. Also, they explain why so many drugs act only on the symptomatic level, without influencing the underlying regulatory network.

- Dynamism and continuous adaptation through bifurcation explain why the system evolves during chronic illness despite administering drugs, which slow down a pathogenic process but perhaps indirectly influence other processes. This could then provoke frailty of other subsystems that de-regulate themselves through time.

- Information and entropy explain why most existing drugs do not give any long-lasting instrument to improve system organization and to keep its entropy low.

- The six levels of systemic organization explain drug pharmacokinetics and pharmacodynamics and clarify the interactions between different drugs. Together with the information and entropy theories, the six levels explain why a clinical case can become very complicated after polytherapy. They supply a systemic frame to study drug reactions not only from a local/physical point of view, but also from an emotional, cognitive, and social point of view. 
- The involvement of the different levels allows us to better target a therapy, and to understand the priorities for treating the subject quickly, effectively, and in the long term. At the same time, they allow the study of chronic illnesses in a prospective way.

\section{Conclusions}

We have explained how cybernetic and complex systems describe the organism in a systemic way. Self-organization, network structure, hierarchical organization, non-linearity, feedback circuits, reactions to information, and the view of drugs as information clarify the existing pharmacological methods. They also offer interesting perspectives for a systemic research, for the development of the next generation drugs and for the development of precision medicine. If we test these hypotheses, we can develop therapies that are compatible with a body systemic organization and low entropy. In our opinion, multi-targeted drugs should be able not only to block a receptor for a limited time but also to act on the remote cause of the pathogenic cascade. Furthermore, those drugs could be administered based on individual variability and personal networks. We believe that our proposed approach could help the development of individualized, precise, and integrated medicine. We are convinced that this new approach will contribute to curing an ever-increasing number of illnesses.

Conflicts of Interest: We do not have any conflict of interest to declare.

Funding sources: This research did not receive any specific grant from funding agencies in the public, commercial, or not-for-profit sectors. 


\section{References:}

[1] Barabási A-L, Gulbahce N, Loscalzo J (2011) Network medicine: a network-based approach to human disease. Nat Rev Genet 12: 56-68. doi:10.1038/nrg2918.

[2] Chan SY, Loscalzo J (2012) The Emerging Paradigm of Network Medicine in the Study of Human Disease. Circ Res 111: 359-74. doi:10.1161/CIRCRESAHA.111.258541.

[3] Goh K-I, Cusick ME, Valle D, Childs B, Vidal M, Barabási A-L (2007) The human disease network. Proc Natl Acad Sci U S A 104: 8685-90. doi:10.1073/pnas.0701361104.

[4] Lu Y-F, Goldstein DB, Angrist M, Cavalleri G (2014) Personalized Medicine and Human Genetic Diversity. Cold Spring Harb Perspect Med 4 :a008581-a008581. doi:10.1101/ cshperspect.a008581.

[5] Ashley EA (2015) The Precision Medicine Initiative. JAMA 313: 2119-20. doi:10.1001/jama. 2015.3595.

[6] (2016) Precision Medicine: Harnessing the Extraordinary Growth in Medical Data for Personalized Diagnosis and Treatment, http://claudiacopeland.com/uploads/3/6/1/4/3614974/ hjno_novdec_2016_precision_medicine.pdf

Accessed december 2016

[7] WHO (2003) WHO | Traditional medicine Strategy, http://www.searo.who.int/entity/ health situation trends/who trm strategy 2014-2023.pdf?ua=1

Accessed 2003

[8] Wiener N (2012) Introduzione alla cibernetica: l'uso umano degli esseri umani. Bollati Boringhieri

[9] Wiener N (1948) Cybernetics: Or control and communication in the animal and the machine. Cambridge: MIT Press

[10] Bateson G (1976) Steps to an Ecology of Mind. Ballantine Nonfiction: Ballantine Books 
[11] Turchin VF (1977) The Phenomenon of Science. New York: Columbia University Press

[12] Telfener U, Casadio L (2003) Sistemica: voci e percorsi nella complessità. Bollati Boringhieri

[13] Malerba M (2017) Occlusione integrata. Youcanprint

[14] Ashby WR (2015) An Introduction to Cybernetics. Martino Fine Books

[15] Licata I, Coniglione F (2015) I gatti di Wiener: riflessioni sistemiche sulla complessità.

Bonanno

[16]Anderson PW (1972) More is different. Science 177:393-6. doi:10.1126/science.177.4047.393.

[17] Bodenschatz E (2009) Complex Systems,

https://www.mpg.de/36885/cpt08_ComplexSystems-basetext.pdf. Accessed 2009

[18] Bettelli O (2004) Modelli per Sistemi Complessi. Brescia, Italy: Starrylink

[19] Bertuglia CS, Vaio F (2011) Complessità e modelli. Un nuovo quadro interpretativo per la modellizzazione nelle scienze della natura e della società. Torino, Italy: Bollati Boringhieri

[20] Plsek PE, Greenhalgh T (2001) The challenge of complexity in health care. BMJ 323:625-8. doi:10.1136/BMJ.323.7313.625.

[21] Gell-Mann M (2002) What is complexity? In: Curzio AQ, Fortis M, editors. Complexity and Industrial Clusters, Physica-Verlag HD p.13-24.

[22] Cannon, WB (1963) The wisdom of the body. W. W. Norton \& Company

[23] Ruelle D (1989) Elements of differentiable dynamics and bifurcation theory. Academic Press

[24] Werndl C (2009) What are the New Implications of Chaos for Unpredictability? The British Journal for the Philosophy of Science 60 (1): 195-220. doi:10.1093/bjps/axn053.

[25] Lorenz EN (1963) Deterministic Nonperiodic Flow. J Atmos Sci 20:130-41. doi: 10.1175/1520-0469(1963)020<0130:DNF>2.0.CO;2.

[26] Prigogine I, Stengers I (1979) La Nouvelle Alliance: Métamorphose de La Science. Paris: Gallimard Editor 
[27] De Robertis D (2005) Le logiche dei Sistemi Complessi: un potenziale per la teoria e la clinica psicoanalitica. Ricerca psicoanalitica 319-330.

[28] Cappelletti P (2014) La complessità e la medicina di laboratorio. La Riv Ital Della Med Di Lab - Ital J Lab Med 10:187-92. doi:10.1007/s13631-014-0074-y.

[29] Casati G (1991) Il Caos. Le leggi del disordine. Milano: Le Scienze S.p.a.

[30] O’Connor, Timothy and Wong HY (2015) Emergent Properties. In: Edward N. Zalta, editor. Stanford Encycl. Philos. Summer 2015, Metaphysics Research Lab, Stanford University

[31] Goldstein J (1999) Emergence as a Construct: History and Issues. Emergence 1:49-72. doi: 10.1207/s15327000em0101_4.

[32] Smith LB, Thelen E (2003) Development as a dynamic system. Opin TRENDS Cogn Sci 7:343. doi:10.1016/S1364-6613(03)00156-6.

[33] Stolorow RD (1995) An intersubjective view of self psychology. Psychoanal Dialogues 5:3939. doi:10.1080/10481889509539077.

[34] Diani S (2018) Are Diseases the Best Possible Response of the Complex Living System to Stimuli? International Journal of History and Philosophy of Medicine 8: 10802.

[35] Prigogine I, Stengers I (1979) La Nouvelle Alliance: Métamorphose de La Science. Paris: Gallimard Editor

[36] Maturana H, Varela F (1987) The Tree of Knowledge: The Biological Roots of Human Understanding. Boston: Shambala

[37] Choi D, Lee TH, Spinelli C, Chennakrishnaiah S, D’Asti E, Rak J (2017) Extracellular vesicle communication pathways as regulatory targets of oncogenic transformation. Semin Cell Dev Biol 67:11-22. doi:10.1016/j.semcdb.2017.01.003.

[38] Subramanian A, Schilling TF (2015) Tendon development and musculoskeletal assembly: emerging roles for the extracellular matrix. Development 142:4191-204. doi:10.1242/dev.114777. 
[39] Dityatev A, Schachner M, Sonderegger P (2010) The dual role of the extracellular matrix in synaptic plasticity and homeostasis. Nat Rev Neurosci 11:735-46. doi:10.1038/nrn2898.

[40] Pieter A (2018) Information. In: Edward N. Zalta, editor. Stanford Encycl. Philos. Fall 2018, Metaphysics Research Lab, Stanford University

[41] Bellavite P (2009) La complessità in medicina: fondamenti di un approccio sistemico-dinamico alla salute, alla patologia e alle terapie integrate. Milano: Tecniche nuove

[42] Vicentini Missoni M (1992) Dal calore all'entropia. Una introduzione alla termodinamica. La Nuova Italia

[43] Wiener N (1950) The human use of human beings. Cybernetics and Society

[44] Prigogine I (1945) Moderation and irreversbile transformation in open systems. Bull. Acad. Roy. Belg. Cl. Sci. 31:600-606.

[45] Zanarini G (1996) Entropia, informazione, complessità. Dipartimento di Fisica: Università di Bologna

[46] Ravasz E, Barabási A-L (2003) Hierarchical organization in complex networks. Phys Rev E 67:026112. doi:10.1103/PhysRevE.67.026112.

[47] Damasio A (2005) Descartes' Error: Emotion, Reason, And the Human Brain. Penguin Group USA

[48] Diani S (2017) Medicina Coerente. Modelli sistemici per una medicina più efficace, umana, individualizzata. Scotts Valley: Createspace

[49] Chen WW, Zhang X, Huang WJ (2016) Role of physical exercise in Alzheimer's disease.

Biomed Reports 4: 403-7. doi:10.3892/br.2016.607.

[50] Hirsch MA, Farley BG (2009) Exercise and neuroplasticity in persons living with

Parkinson's disease. Eur J Phys Rehabil Med 45: 215-29. 
[51] Nithianantharajah J, Hannan AJ (2006) Enriched environments, experience-dependent plasticity and disorders of the nervous system. Nat Rev Neurosci 7: 697-709. doi:10.1038/ nrn1970.

[52] Shulman LM, Katzel LI, Ivey FM, Sorkin JD, Favors K, Anderson KE, et al (2013) Randomized clinical trial of 3 types of physical exercise for patients with Parkinson disease. JAMA Neurol 70: 183-90. doi:10.1001/jamaneurol.2013.646.

[53] Ahlskog JE, Geda YE, Graff-Radford NR, Petersen RC (2011). Physical Exercise as a Preventive or Disease-Modifying Treatment of Dementia and Brain Aging. Mayo Clin Proc 86:876-84. doi:10.4065/mcp.2011.0252.

[54] Erickson KI, Voss MW, Prakash RS, Basak C, Szabo A, Chaddock L, et al (2011) Exercise training increases size of hippocampus and improves memory. Proc Natl Acad Sci USA 108: 3017-22. doi:10.1073/pnas.1015950108.

[55] Sink KM, Espeland MA, Castro CM, Church T, Cohen R, Dodson JA, et al (2015) Effect of a 24-Month Physical Activity Intervention vs Health Education on Cognitive Outcomes in Sedentary Older Adults. JAMA 314: 781-90. doi:10.1001/jama.2015.9617.

[56] Elwood P, Galante J, Pickering J, Palmer S, Bayer A, Ben-Shlomo Y, et al (2013) Healthy lifestyles reduce the incidence of chronic diseases and dementia: evidence from the Caerphilly cohort study. PLoS One 8 :e81877. doi:10.1371/journal.pone.0081877.

[57] Zigmond MJ, Cameron JL, Leak RK, Mirnics K, Russell VA, Smeyne RJ, et al (2009). Triggering endogenous neuroprotective processes through exercise in models of dopamine deficiency. Parkinsonism Relat Disord 15: S42-5. doi:10.1016/S1353-8020(09)70778-3. [58] Oliff HS, Berchtold NC, Isackson P, Cotman CW (1998) Exercise-induced regulation of brain-derived neurotrophic factor (BDNF) transcripts in the rat hippocampus. Brain Res Mol Brain Res 61: 147-53. 
[59] Marais L, Stein DJ, Daniels WMU (2009) Exercise increases BDNF levels in the striatum and decreases depressive-like behavior in chronically stressed rats. Metab Brain Dis 24: 58797. doi:10.1007/s11011-009-9157-2.

[60] Lombardo MP (2008) Access to Mutualistic Endosymbiotic Microbes: An Underappreciated Benefit of Group Living. Behav Ecol Sociobiol 62: 479-97. doi: $10.2307 / 25511719$.

[61] Troyer K. Microbes, herbivory and the evolution of social behavior (1984) J Theor Biol 106: 157-69. doi:10.1016/0022-5193(84)90016-X.

[62] Grenham S, Clarke G, Cryan JF, Dinan TG (2011) Brain?Gut?Microbe Communication in Health and Disease. Front Physiol 2: 94. doi:10.3389/fphys.2011.00094.

[63] Duerkop BA, Vaishnava S, Hooper LV (2009) Immune Responses to the Microbiota at the Intestinal Mucosal Surface. Immunity 31: 368-76. doi:10.1016/j.immuni.2009.08.009. [64] Forsythe P, Bienenstock J (2010) Immunomodulation by Commensal and Probiotic Bacteria. Immunol Invest 39: 429-48. doi:10.3109/08820131003667978.

[65] Bravo JA, Forsythe P, Chew MV, Escaravage E, Savignac HM, Dinan TG, et al (2011) Ingestion of Lactobacillus strain regulates emotional behavior and central GABA receptor expression in a mouse via the vagus nerve. Proc Natl Acad Sci 108: 16050-5. doi:10.1073/pnas. 1102999108.

[66] Desbonnet L, Garrett L, Clarke G, Bienenstock J, Dinan TG (2008) The probiotic Bifidobacteria infantis: An assessment of potential antidepressant properties in the rat. $\mathrm{J}$ Psychiatr Res 43: 164-74. doi:10.1016/j.jpsychires.2008.03.009.

[67] Porges SW (2003) The Polyvagal Theory: phylogenetic contributions to social behavior. Physiol Behav 79: 503-13. 
[68] Mayer EA (2011) Gut feelings: the emerging biology of gut-brain communication. Nat Rev Neurosci 12: 453-66. doi:10.1038/nrn3071.

[69] Craig AD (2009) A rat is not a monkey is not a human: comment on Mogil. Nat Rev Neurosci 10: 466-466. doi:10.1038/nrn2606-c1.

[70] Mayer EA, Saper CE (2000) The biological basis for body interactions. AmsterdamOxford:Elsevier

[71] Bailey MT (2016) Psychological Stress, Immunity, and the Effects on Indigenous Microflora. Adv Exp Med Biol 874: 225-46. doi:10.1007/978-3-319-20215-0_11.

[72] Faresjö M (2015) The Link between Psychological Stress and Autoimmune Response in Children. Crit Rev Immunol 35: 117-34.

[73] Nicolaides NC, Kyratzi E, Lamprokostopoulou A, Chrousos GP, Charmandari E (2015) Stress, the Stress System and the Role of Glucocorticoids. Neuroimmunomodulation 22: 6-19. doi:10.1159/000362736.

[74] Edwards R, Peet M, Shay J, Horrobin D (1998) Omega-3 polyunsaturated fatty acid levels in the diet and in red blood cell membranes of depressed patients. J Affect Disord 48: 149-55. [75] Umetani K, Singer DH, McCraty R, Atkinson M (1998) Twenty-four-hour time domain heart rate variability and heart rate: relations to age and gender over nine decades. J Am Coll Cardiol 31: 593-601.

[76] Frasure-Smith N, Lespérance F, Talajic M (1995) Depression and 18-month prognosis after myocardial infarction. Circulation 91: 999-1005.

[77] Doidge N (2016) The Brain's Way of Healing: Stories of Remarkable Recoveries and Discoveries. Penguin 
[78] Delehant M (2005) Emergent properties and the context objection to reduction. Biology and Philosophy 20: 715-734.

[79] Yehuda R, Halligan SL, Grossman R (2001) Childhood trauma and risk for PTSD: relationship to intergenerational effects of trauma, parental PTSD, and cortisol excretion. Dev Psychopathol 13: 733-53.

[80] Elzinga BM, Schmahl CG, Vermetten E, van Dyck R, Bremner JD (2003) Higher Cortisol Levels Following Exposure to Traumatic Reminders in Abuse-Related PTSD.

Neuropsychopharmacology 28: 1656-65. doi:10.1038/sj.npp.1300226.

[81] Herane-Vives A, Fischer S, de Angel V, Wise T, Cheung E, Chua K-C, et al (2018) Elevated fingernail cortisol levels in major depressive episodes. Psychoneuroendocrinology 88: 17-23. doi:10.1016/j.psyneuen.2017.10.026.

[82] Bizzarri M (1999) La mente e il cancro. Milano: Frontiera

[83] Luthe, W (1973) Autogenic therapy: Treatment with autogenic neutralization. New York: Grune and Stratton.

[84] Kang E-H, Park J-E, Chung C-S, Yu B-H (2009) Effect of biofeedback-assisted autogenic training on headache activity and mood states in Korean female migraine patients. J Korean Med Sci 24: 936-40. doi:10.3346/jkms.2009.24.5.936.

[85] Hidderley M, Holt M (2004) A pilot randomized trial assessing the effects of autogenic training in early stage cancer patients in relation to psychological status and immune system responses. Eur J Oncol Nurs 8: 61-5. doi:10.1016/j.ejon.2003.09.003.

[86] Miu AC, Heilman RM, Miclea M (2009) Reduced heart rate variability and vagal tone in anxiety: Trait versus state, and the effects of autogenic training. Auton Neurosci 145: 99-103. doi: 10.1016/j.autneu.2008.11.010. 
[87] Seo E, Kim S (2019) Effect of Autogenic Training for Stress Response: A Systematic Review and Meta-Analysis. J Korean Acad Nurs. Aug;49(4):361-374. doi: 10.4040/jkan.2019.49.4.361.

[88] Shapiro S, Lehrer PM (1980) Psychophysiological effects of autogenic training and progressive relaxation. Biofeedback Self Regul 5: 249-55. 